\title{
Analysis of Traffic Conditions in Urban Region Based on Data from Fixed Detectors
}

\author{
Shidong Liang, Shuzhi Zhao, Minghui Ma, and Huasheng Liu \\ College of Transportation, Jilin University, Changchun 130022, China \\ Correspondence should be addressed to Huasheng Liu; liuhuasheng521@163.com
}

Received 2 June 2015; Revised 10 August 2015; Accepted 11 August 2015

Academic Editor: Lawrence P. Horwitz

Copyright (C) 2015 Shidong Liang et al. This is an open access article distributed under the Creative Commons Attribution License, which permits unrestricted use, distribution, and reproduction in any medium, provided the original work is properly cited.

To evaluate traffic conditions in an urban region, the average travel speed in the street network and the average traffic flow per lane are selected as indexes. The results for identifying traffic conditions are obtained using macroscopic fundamental diagram. Because of the difficulty of collecting the traffic parameter of travel speed from widely distributed fixed detectors directly, in this paper, the relationship between average travel speed and queue lengths at signalized intersections within the urban area is established based on an equivalent assumption. Finally, the average travel speed estimation model proposed in this paper is tested with microscopic simulation platform, and the results showed that the average travel speed estimation model performed well with satisfactory accuracy. In addition, the best performance of the network efficiency can be identified based on the reflections of macroscopic fundamental diagram, and the evaluation system is simple enough for application in engineering. Therefore, the result is instructive both for generating traffic control strategies and for making route choices.

\section{Introduction}

Traffic conditions analysis has long been studied. As an index of traffic performance, the ability to estimate the traffic conditions is crucial to both traffic control and traffic guidance.

In early research, scholars focused on incident identification. Essentially, they described what was happening on the street by evaluating the real-time traffic flow information or the time occupancy data gathered from fixed detectors that were placed upstream and downstream $[1,2]$ or in adjacent lanes [3]. Some artificial intelligence algorithms were also introduced into the research [4]. Although such methods performed well in analyzing the traffic flow for freeway and arterial streets, these methods seem insufficient to evaluate urban traffic conditions because it is difficult to describe a complex urban system based on local traffic parameters.

As traffic demand increases, the occurrence of frequent traffic jams and congestions becomes a major issue. Therefore, the result of whether traffic incident occurred cannot meet the demands of traffic managers or drivers. Traffic conditions evaluation methods can be classified into two types: data mining methods based on the located traffic parameters; macroscopic traffic flow parameters estimation based on advanced equipment.

Macroscopic traffic flow parameters estimations are based on information from sophisticated equipment. The data from GPS [5, 6], video [7], and phone [8] is collected and processed to obtain these macroscopic traffic flow parameters, such as travel time, queue length, and traffic density. To further improve the quality of the data collected, some scholars evaluated the traffic conditions with multisource data of the located traffic flow parameters using the fusion method [9, 10]. However, the maturity of these techniques as well as the sample size has great influence on the accuracy of the parameters estimations. Also, because funding is limited, not all the facilities can be equipped. We would like to express that the proposed model is operable, because the data from fixed detectors of traditional signal control systems, such as SCOOT system, can be input for the proposed model. Specifically, the fixed detectors of traditional signal control systems have been widely placed in urban street, so that the model input data can be easily accessed. It is not necessary to lay more new detectors on the street. Therefore, our work is meaningful in the implement in urban network. 


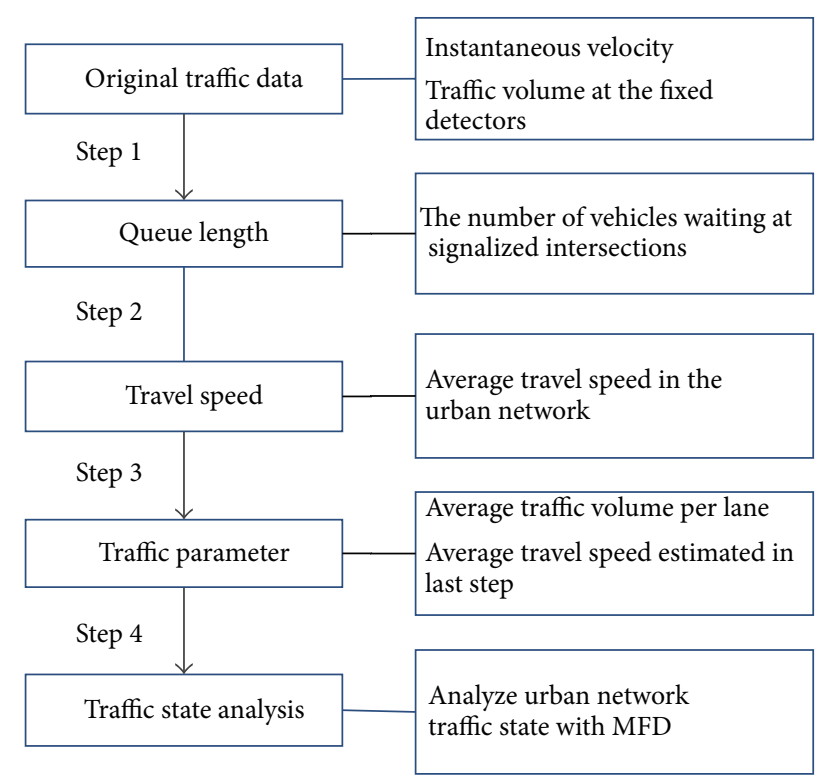

Figure 1: Flow chart of traffic state analysis.

Above all, the traffic flow as detected by fixed detectors is reliable data. The technology of a loop detector that counts the number of vehicles in a time interval is mature enough to be used. However, traffic flow merely cannot accurately represent the traffic conditions. Whether the urban region network is jammed or is free flowing, the traffic flows maintain a relatively low level. Therefore, another index is required to fix the defect. Travel speed can reflect the running efficiency of current traffic flow. As mentioned above, traffic flow parameters have been obtained using mobile detectors in the past, though the accuracy of the data is limited by the number of detectors used. Therefore, it is important to obtain travel speed data using fixed detectors, which are widely spread in an urban region network.

To establish the relationship between travel speed and located traffic parameter, the data about the queue length at a signalized intersection is introduced as a middle stage. The methods of queue length estimation are quite mature. However, as the definition of queue length is different from the traditional meaning, the results should be modeled for a specific situation instead of being directly input for an average travel speed estimation model. As the traffic conditions result is meaningless if traffic conditions are uneven in the urban region under research, we select a traffic control subarea, and the traffic flow is relatively balanced in each direction.

Figure 1 here presents the process from original data collected by fixed detectors to traffic state analysis. The middle two steps, queue length calculation method and travel speed estimation model, are the major contributions of this paper. As shown in Figure 1, the original data collected by fixed detectors is insufficient to obtain traffic parameters of traffic flow in urban network. Therefore, we estimate the traffic parameter required indirectly.

This paper is organized as follows. The average travel speed definition is clarified in Section 2, followed by the estimation model. Section 3 presents the difference between traditional queue length and the required queue length for this proposed speed estimation model and also presents the formula and calculation method of queue length at a signalized intersection. In Section 4, the performance of average travel speed estimation model is tested, followed by an application for the traffic conditions analysis method. The conclusion and some limitations are presented in Section 5.

\section{Average Travel Speed Estimation Model}

As an important traffic index, travel speed is well understood by travelers. They can easily estimate the time required to travel from origin to destination. To a certain extent, the travel speed represents the current traffic condition in the detected area. When the traffic is heavy, vehicles running in urban streets interact with each other frequently and the waiting time at signalized intersections is longer, which decreases the average travel speed in comparison to light traffic flow conditions.

The travel speed proposed in this paper is defined as the average travel speed of all the vehicles running in the detected urban region during a certain time interval. From the definition, it is obvious that the travel speed should be detected over some distance. In practice, the data of travel speed is collected using technological means such as GPS and video. Though the fixed detectors seem insufficient to get this traffic index directly, they are widely applied in the urban road network and perform well in terms of stability and accuracy. As the fixed detectors are expensive and indispensable in some traditional signal control systems (such as the SCOOT system), it is meaningful to estimate travel speed using data from fixed detectors.

2.1. An Equivalent Assumption. In order to obtain the average travel speed in an urban region network indirectly, the relationship between average travel speed and queue lengths at signalized intersections is formulated. For a single vehicle, travel time $t_{\text {travel }}$ can be expressed as

$$
t_{\text {travel }}=\frac{l_{\text {travel }}}{v_{\text {travel }}}
$$

where $l_{\text {travel }}$ is equal to the distance of the vehicle travelling in a time interval and $v_{\text {travel }}$ refers to its travel speed. The travel time $t_{\text {travel }}$ includes two parts that are the time running $t_{\text {run }}$ on the street and the delay $t_{\text {delay }}$ at signalized intersection. So, the travel time also can be written as

$$
t_{\text {travel }}=t_{\text {run }}+t_{\text {delay }} \text {. }
$$

We assume that the vehicles on an urban network include two conditions: running with free flow speed $v_{\text {free }}$ and waiting at a signalized intersection. $t_{\text {run }}$ and $t_{\text {delay }}$ can be formulated, respectively, as

$$
\begin{gathered}
t_{\text {run }}=\frac{l_{\text {travelling }}}{v_{\text {free }}}, \\
t_{\text {delay }}=n_{\text {travel }} \cdot \bar{D},
\end{gathered}
$$


where $\bar{D}$ refers to the average delay of signalized intersection and $n_{\text {travel }}$ means number of intersections in the traveling track of the vehicle. The value of $n_{\text {travel }}$ can obtained by

$$
n_{\text {travel }}=\frac{l_{\text {travel }}}{\overline{l_{\text {dis }}}},
$$

where $\overline{l_{\text {dis }}}$ is the average distance between neighboring intersections.

From the five equations above, the average travel speed of the vehicles in an urban region can be obtained by the formula, with eliminating travel distance of single vehicle $l_{\text {travel }}$ :

$$
\overline{v_{\text {travel }}}=\frac{v_{\text {free }} \cdot \overline{l_{\text {dis }}}}{\overline{l_{\text {dis }}}+v_{\text {free }} \cdot \bar{D}} .
$$

In (6), all the variables can be measured except the average vehicle delay at intersections. To calculate this traffic variable, we should make a crucial equivalent assumption here. In practice, the traffic flow is not continuous because of signal control at intersections. However, the traffic condition of an urban region is considered stable in a certain interval. Therefore, we should transform the traffic flow into an equivalent stable continuous flow. As the number of released vehicles is conservative at intersection in a signal cycle and the vehicles are allowed to pass the intersection all the time, the entrance lanes should be narrowed down. We define the narrowed lanes as equivalent lanes. For each traffic flow direction $i$, the number of equivalent lanes $n_{i, \text { eq }}$ can be obtained by signal timing parameters of green-light time $g_{i}$, signal cycle $C$, saturated flow $S$, and the number of real entrance lanes $n_{i \text {,real }}$ with the formulation

$$
\begin{aligned}
\frac{g_{i} \cdot n_{i, \text { real }} \cdot S}{C} & =\frac{C \cdot n_{i, \mathrm{eq}} \cdot S}{C} \\
& \Longrightarrow n_{i, \text { eq }}=n_{i, \text { real }} \cdot \frac{g_{i}}{C} .
\end{aligned}
$$

According to principle of vehicles conservation, the number of queueing at signalized intersection is equal. The relationship between real queue length $l_{i \text {,real }}$ at entrance lanes and equivalent queue length $l_{i \text {,eq }}$ at equivalent lanes can be expressed with

$$
\begin{aligned}
l_{i, \text { eq }} \cdot n_{i, \text { eq }} \cdot k_{J} & =l_{i, \text { real }} \cdot n_{i, \text { real }} \cdot k_{J} \\
& \Longrightarrow l_{i, \text { eq }}=l_{i, \text { real }} \cdot \frac{n_{i, \text { real }}}{n_{i, \text { eq }}},
\end{aligned}
$$

where $k_{J}$ refers to jam density per lane.

\subsection{Relation between Average Travel Speed and Queue} Lengths. In a short time interval, the traffic condition remains stable. Hence, the number of queuing vehicles is a dynamic equilibrium, which means the value of equivalent queue length $l_{i \text {,eq }}$ is stable.

Figure 2 presents an example of process how the vehicles wait in the queue line and leave the intersection. There are five

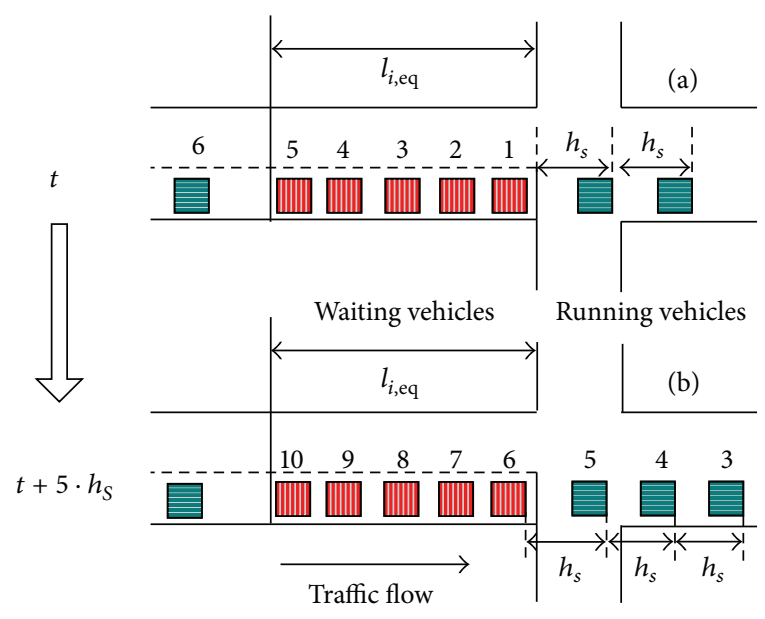

FIgURE 2: Process of vehicles passing intersection.

vehicles in the queue line. The vehicle numbered five locates at the rear of the equivalent queue at time $t$ and cannot pass the stop line until the front four vehicles are released into the intersection with a saturated flow rate $\left(h_{S}\right.$ means saturated headway). When the number five vehicle passes the stop line at time $t+5 \cdot h_{S}$, the vehicle numbered ten joins the queue immediately. Like the number five vehicle, the vehicle at end of the queue has to leave the intersection after $5 \cdot h_{S}$. In a similar fashion, every vehicle heading in this direction delays the same time $\overline{d_{i}}$ during this time period, which depends on the queue length and the saturated headway:

$$
\overline{d_{i}}=k_{J} \cdot h_{S} \cdot l_{i, \mathrm{eq}} .
$$

Substitute (8) into (9):

$$
\overline{d_{i}}=k_{J} \cdot h_{S} \cdot l_{i, \text { real }} \frac{C}{g_{i}} .
$$

To achieve the total delay of this direction $d_{i, \text { total }}$, the number of queued vehicles $N_{i}$ during this time period should be calculated:

$$
\begin{aligned}
N_{i} & =k_{J} \cdot n_{i, \text { eq }} \cdot l_{i, \text { eq }}=k_{J} \cdot n_{i, \text { real }} \cdot l_{i, \text { real }}, \\
d_{i, \text { total }} & =\overline{d_{i}} \cdot N_{i}=k_{J}^{2} \cdot l_{i, \text { real }}^{2} \cdot n_{i, \text { real }} \cdot h_{S} \cdot \frac{C}{g_{i}} .
\end{aligned}
$$

There are $M$ intersections in the urban region. The average delay $\overline{D_{m \text {,aver }}}$ for an intersection $m(m \in\{1,2, \ldots, M\})$ can be obtained by calculating the total vehicle delay of all the directions divided by the total number of queued vehicles at the intersection:

$$
\overline{D_{m, \text { aver }}}=\frac{\sum d_{i, \text { total }}}{\sum N_{i}}=\frac{k_{J} \cdot h_{S} \cdot C}{\sum l_{i, \text { real }} \cdot n_{i, \text { real }}} \cdot \sum \frac{l_{i, \text { real }}^{2} \cdot n_{i, \text { real }}}{g_{i}} .
$$

Similarly, the average vehicle delay $\bar{D}$ (mentioned in (6)) of all the intersections can be achieved by calculating the total vehicle delay of all the intersections divided by the total 
queued vehicles in the urban area. Substituting $\bar{D}$ into (6), the average travel speed in the urban region can be expressed by

$$
\overline{v_{\text {travel }}}=\frac{\overline{l_{\text {dis }}} \cdot v_{\text {free }} \cdot \sum_{m=1}^{M} N_{m}}{\overline{l_{\text {dis }}} \cdot \sum_{m=1}^{M} N_{m}+v_{\text {free }} \cdot \sum_{m=1}^{M} \overline{D_{m \text {,aver }}} \cdot N_{m}} .
$$

Equations (12) and (13) establish a relationship between average travel speed and queue lengths at intersections. The following section presents the method used to measure the queue length required in this proposed model.

\section{Queue Length Calculation Method}

Because it is an important input parameter for the average travel speed estimation model, the accuracy of the queue length calculation directly impacts the performance of the traffic conditions evaluation. Thus, it is necessary to study the method of calculating the estimation of queue length required for (12).

3.1. Queue Length during Unsaturated Traffic Flow. Queue length estimation has been a topic of study for a long time. Many scholars dedicated themselves to this topic, and their proposed models can be classified into three types: inputoutput accumulation curve methods, statistical methods, and the shockwave theory based method.

Shockwave theory is a powerful tool in queue length estimation. It was proposed by Lighthill and Whitham (1955) [11] and Richards (1956) [12] to describe the queueing process for uninterrupted traffic flow on a freeway. Later, Michalopoulos and Stephanopoulos (1977) [13] expanded the theory for continuum traffic flow at signalized intersections. Some scholars (Liu et al. 2009) [14] were more ambitious in estimating the real-time queue length under oversaturated flow. Therefore, these traditional models can nicely estimate queue length at signalized intersections. However, the outcome value cannot be used directly in this proposed model. The traditional queue length refers to the distance between the rear of the queue and the stop line at a given moment. There are two differences between the traditional and our required queue length: (1) the required queue length for this model means the length of the immobile vehicles instead of the queue back; (2) the required queue length for this model implies the average queue length during a time interval instead of at a given moment.

Figure 3 presents the process of vehicles arriving and leaving at signalized intersection. Real-time queue lengths mean that the queue backs in each moment. Area $\mathrm{A}$ and area $B$ represent the summation of the queue lengths. However, as the vehicles of area B have moved during the green-light time, this part should be cut off. Otherwise, the delay will be overestimated in Section 2.

In traditional research, the queue lengths $l_{1}, l_{2}, \ldots$, and $l_{\max }$ can be obtained (see Liu et al. 2009) [14]. The velocity of traffic flow starting wave $w$ can also be calculated by shockwave theory:

$$
w=\frac{\Delta q}{\Delta k}
$$

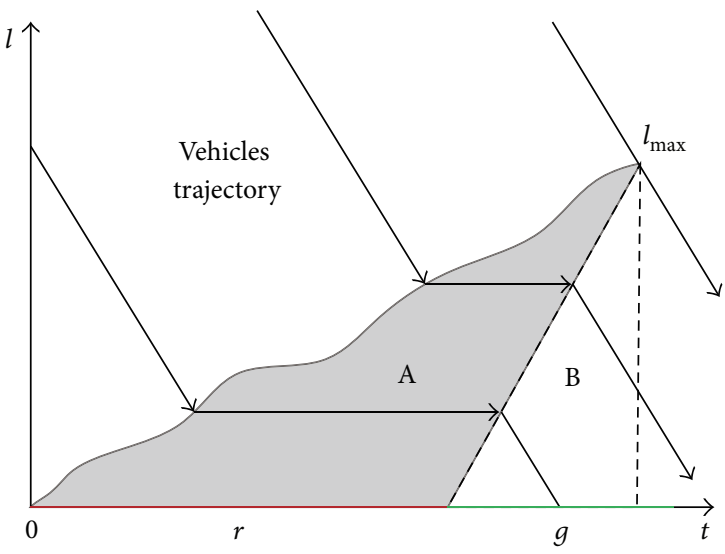

Figure 3: Process of vehicles aggregating and releasing.

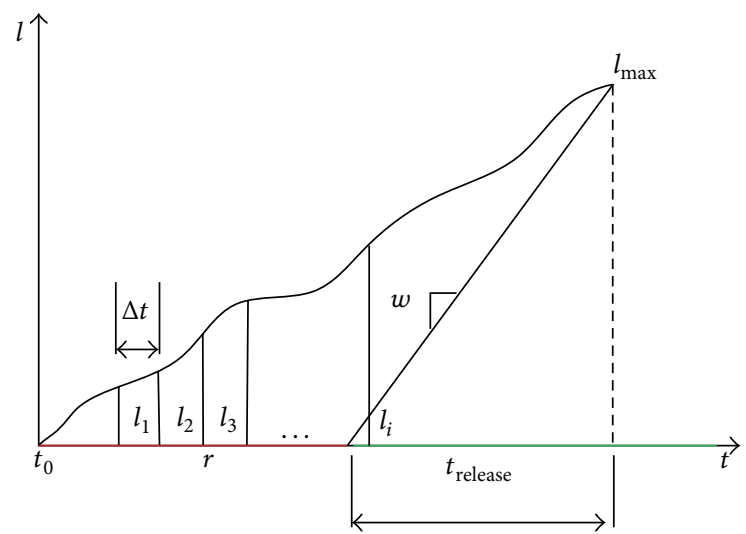

FIGURE 4: General analysis of queue at signalized intersection.

Further, the time of $t_{\text {release }}$ can be achieved by $w$ and $l_{\max }$ with the formula of

$$
t_{\text {release }}=\frac{l_{\max }}{w} .
$$

According to Figures 3 and 4, when the traffic flow is unsaturated all the vehicles leave intersection in a signal cycle; the area of $\mathrm{B}$ is then expressed by

$$
S_{\mathrm{B}}=\frac{1}{2} \cdot t_{\text {release }} \cdot l_{\max } \cdot
$$

In a signal cycle, the general average queue length $l_{i \text {,real }}$ required in (12) can be written as

$$
l_{i \text {,real }}=\frac{\Delta t \cdot \sum l_{i}-S_{\mathrm{B}}}{C}=\frac{2 \cdot \Delta t \cdot \sum l_{i}-t_{\text {release }} \cdot l_{\text {max }}}{2 \cdot C} .
$$

3.2. Queue Length during Saturated Traffic Flow. However, (17) is only suitable in an unsaturated traffic flow situation. When traffic flow turns to saturated, two factors should be considered: (1) the time of maximum queue length 


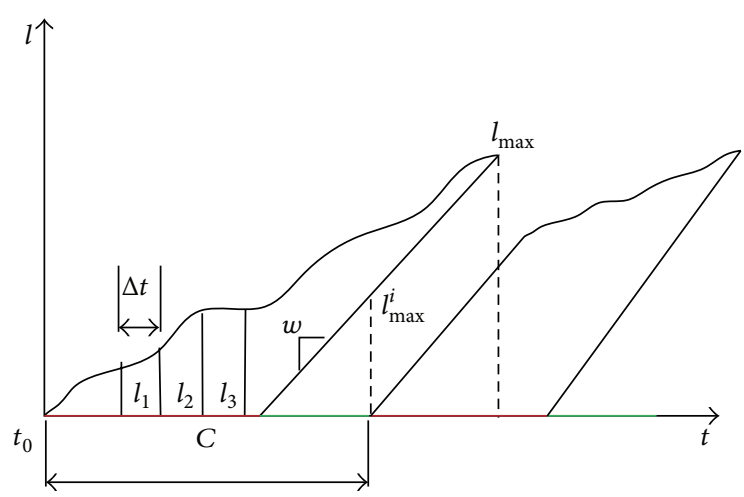

FIgURE 5: Analysis of queue at signalized intersection under saturated traffic flow.

occurrence is beyond the current signal cycle, which affects the method of calculating the area of $\mathrm{B}$; $(2)$ as the time of maximum queue length occurrence occurs in following signal cycle, the queue length calculation method is different from (17). Figure 5 represents the affecting mechanism by saturated traffic flow.

Firstly, the area of B is determined by $l_{\max }^{\prime}$ and green-light time $g$ instead of $l_{\max }$ and $t_{\text {release }}$ with the formula

$$
S_{\mathrm{B}}=\frac{1}{2} \cdot g \cdot l_{\max }^{\prime}=\frac{1}{2} \cdot g^{2} \cdot w
$$

Secondly, in the following cycle, not only the area of B but also the rhomboid area between $l_{\max }^{\prime}$ and $l_{\max }$ should cut off, which can be expressed by

$$
S_{\text {rho }}=\left(\frac{l_{\max }}{w}-g\right) \cdot l_{\max }^{\prime}=\frac{g \cdot l_{\max }}{w}-g^{2} \cdot w .
$$

With respect to the oversaturated traffic flow, the vehicles have to stop $x$ times at signalized intersections. The rhomboid areas $S_{\text {rho }}^{x}$ include several parts, which are $x-1$ similar rhomboid areas just between the starting time of the green light and ending time of the red light in a current signal cycle and one rhomboid area mentioned in (19). Therefore, the rhomboid areas cut-off can be written as

$$
\begin{aligned}
S_{\text {rho }}^{x} & =(x-1) \cdot\left(l_{\max }^{\prime} \cdot C\right)+S_{\text {rho }} \\
& =g \cdot w \cdot C \cdot(x-1)+\frac{g \cdot l_{\max }}{w}-g^{2} \cdot w .
\end{aligned}
$$

The formulas of (16), (17), (18), (19), and (20) describe a method to transform traditional queue back and vehicle stop times into the queue length required by the proposed average speed estimation model.

Now, we obtain the average travel speed indirectly by the method presented in Sections 2 and 3. Also, the average traffic flow per lane can be easily detected by loop detectors. Therefore, a data mining method should be borrowed to analyze the traffic conditions of urban regions, which is introduced in Section 4.
TABLE 1: Input traffic flow for each endpoint of streets.

\begin{tabular}{lllllll}
\hline Time (h) & $0-2$ & $2-3$ & $3-4$ & $4-5$ & $5-6$ & $6-7$ \\
\hline Traffic flow (veh/h) & 603 & 684 & 756 & 803 & 858 & 905 \\
\hline
\end{tabular}

TABLE 2: Random seed setting for simulation.

\begin{tabular}{lccccc}
\hline Simulation times & 1 & 2 & 3 & 4 & 5 \\
\hline Random seed & 42 & 56 & 68 & 73 & 89 \\
\hline
\end{tabular}

\section{Implementation}

4.1. Implementation Design. The proposed method analyzes the urban region traffic conditions using macroscopic fundamental diagram based on the indexes of average travel speed and average traffic flow per lane. Because the data for average travel speed is obtained using the estimation model proposed in Sections 2 and 3, it is necessary to verify the performance of the average travel speed estimation model. Further, the macroscopic fundamental diagram is formulated and the meaning of the result is also discussed. We select the micro simulation soft VISSIM as the test platform.

The urban region to be tested consists of six streets in Changchun City and nine signalized intersections. Figure 6(a) shows the map of urban region selected for the test (including four arterial streets and two secondary roads). Figure 6(b) presents the detectors which are located on the network. The detectors are located on the upstream of the road between neighboring intersections.

The simulation environment is designed as follows. To obtain a uniform traffic condition in an urban region for testing, nine intersections are incorporated into a traffic control subarea. The total time for one simulation time is seven hours. The first hour is useless except for ensuring that the network is filled with vehicles. During the following six hours, the traffic flow variables inputs are shown in Table 1. The traffic flow gradually increasing ensures that all traffic conditions occurred in the urban region network, and the data collected is divided into six groups according to the differing input traffic flow. The output from micro simulation software incorporates the traffic flow per lane and the queue length and stops of each direction at intersections, which are the input variables required by proposed average speed estimation model. Also the average travel speed should be calculated and compared with the estimation values to test the performance of the proposed model.

In the simulation software, random seeds influence the results of vehicle occurrences. Therefore, to insure the reliability of the testing result and weaken the influence of randomness, we ran the micro simulation software five times with different random seeds, which are shown in Table 2.

4.2. Test of the Speed Estimation Model. The input variables for the proposed model come from the output of simulation software, including queue lengths and stop times. The implementation of the average speed calculation relies on MATLAB programs. In addition, the software output should include the real average travel speed to compare with 


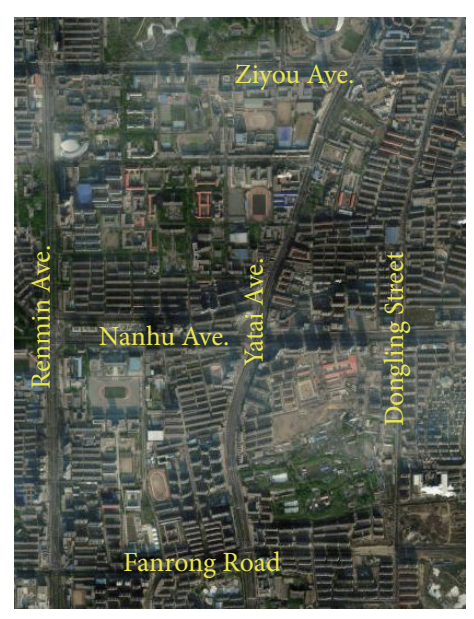

(a)

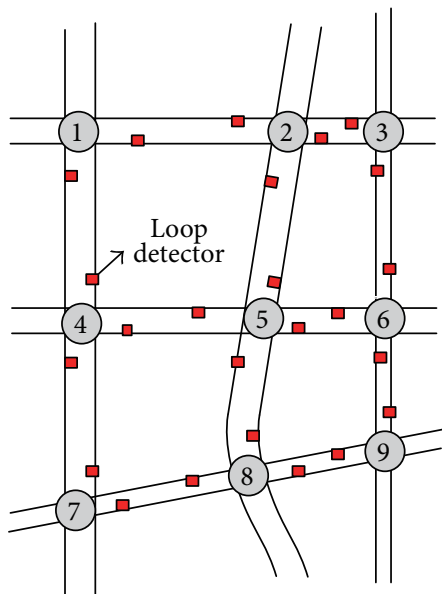

(b)

FIgURE 6: (a) Test field; (b) loop detectors layout.

TABLE 3: Results for five simulation tests.

\begin{tabular}{lcc}
\hline $\begin{array}{l}\text { Simulation } \\
\text { times }\end{array}$ & $\begin{array}{c}\text { Average relative } \\
\text { errors }(\%)\end{array}$ & $\begin{array}{c}\text { Average absolute } \\
\text { errors }(\mathrm{km} / \mathrm{h})\end{array}$ \\
\hline 1 & 3.19 & 0.74 \\
2 & 2.33 & 0.54 \\
3 & 2.82 & 0.65 \\
4 & 3.26 & 0.75 \\
5 & 3.12 & 0.72 \\
\hline
\end{tabular}

the estimation values in order to verify accuracy of proposed model.

First, the average speed data is divided into six groups according to the difference of input for traffic flow. Figure 7 presents the results of the estimation model performance. For each group, the abscissa value of a sample point refers to real average travel speed in a time interval, and its ordinate value means the estimated average travel speed. Therefore, if the sample point locates above the oblique line, it implies overestimation of the speed. Oppositely, it means underestimation of the speed. In Figure 7, most of the samples points are around the oblique line, indicating that the proposed models perform well in average speed estimation in an urban region.

More exactly, Table 3 presents the average relative errors and average absolute errors for five repetitions of the experiments with different random seeds.

Figure 7 and Table 3 show the performance of the estimation model through graphic and tabular forms, respectively. In Figure 7 the percentage of relative error fluctuates between 0 and $10 \%$, and most of the relative errors are less than $5 \%$ exactly. This level of accuracy justifies the application of our traffic conditions analysis model. Further, we ran the software five times with different random seeds. The total results for each simulation are shown in Table 3, including average relative errors and average absolute errors. The average absolute errors and average relative errors are relatively low, about $0.7 \mathrm{~km} / \mathrm{h}$ and $3 \%$, respectively. Therefore, the estimation model is reliable enough to support input data to traffic conditions analysis with acceptable error range.

\subsection{Applications for This Traffic Conditions Analysis Method.}

To test the traffic conditions analysis method, we should build themacroscopic fundamental diagram and urban region network. Figure 8 presents the relationship between average travel speed and the average traffic flow per lane. For each sample, its abscissa value refers to the average traffic flow; the ordinate value means the estimated average travel speed in a time interval.

In Figure 8, the relationship between average travel speed and average traffic flow tends to be a parabolic shape, which is similar to the experimental conclusion proposed by Daganzo and Geroliminis (2008) [15, 16]. During a free flow period, the traffic flow per lane is relatively low and the average travel speed maintains a high level, which leads to significant vehicle randomness. The dispersed distribution of sample points in Figure 8 reflects this randomness. With increasing traffic flow, vehicles have fewer opportunities to change lanes or overtake other vehicles and the speed differences decrease. Therefore, the sample points in Figure 8 are relatively concentrated under these conditions. The meaning of Figure 8 and the character of traffic flow are seen in the mapped relationship. As the urban region is a traffic control subarea, although the input traffic flow increases, the vehicles flowing into the region network will not increase infinitely. Therefore, the conditions will not be worse when traffic flow reaches a traffic jam level. In practice, traffic managers are more concerned with the traffic conditions of an urban sensitive area during a peak period and they need a method to deal with peak periods. The best operation state neither wastes the traffic network capacity nor decreases traffic operation efficiency due to a traffic jam. As shown in Figure 8, the ordinate value of vertex of parabola refers to the maximum traffic volume of the urban network, and the slope of the dotted line means traffic density which reflects the capacity of the urban network. 


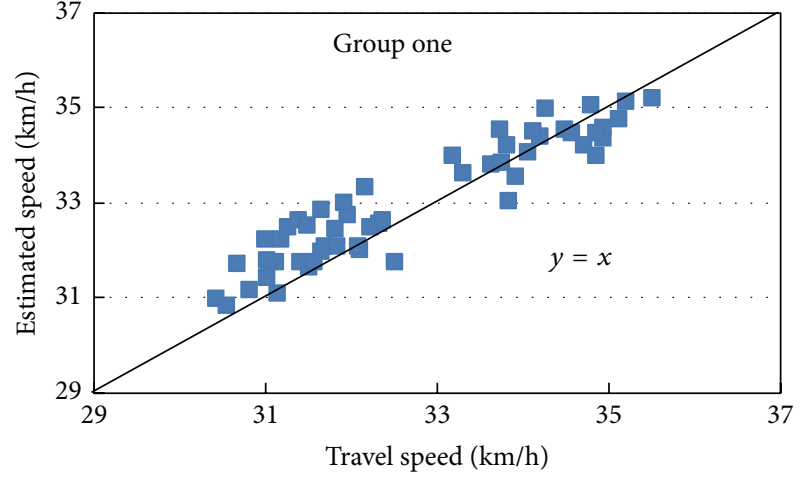

(a)

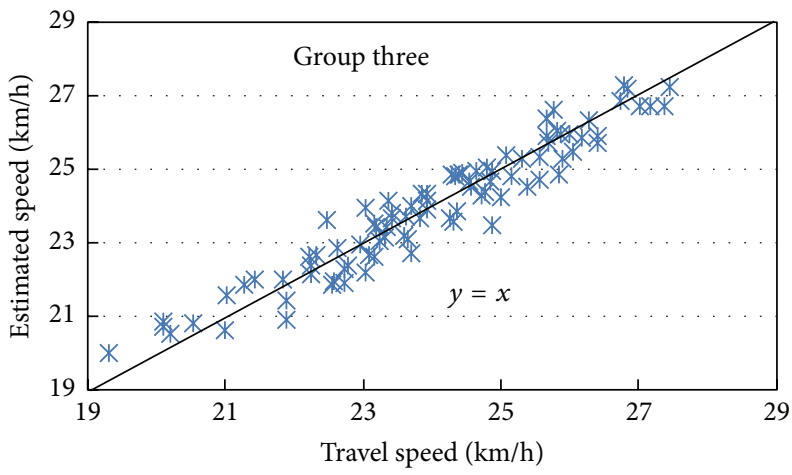

(c)

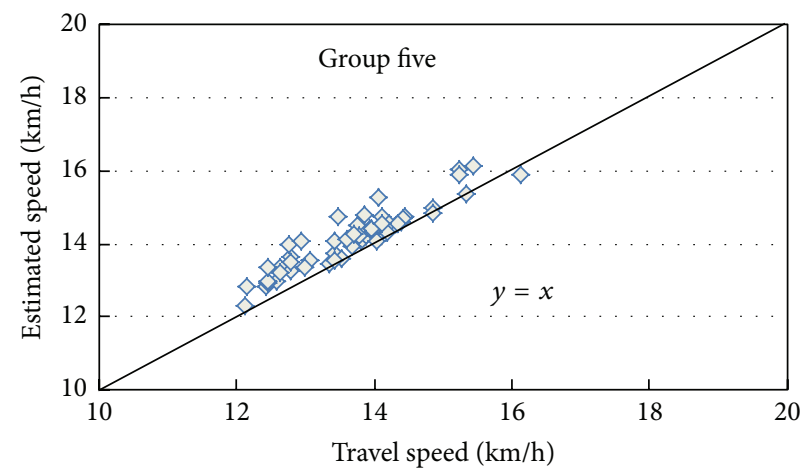

(e)

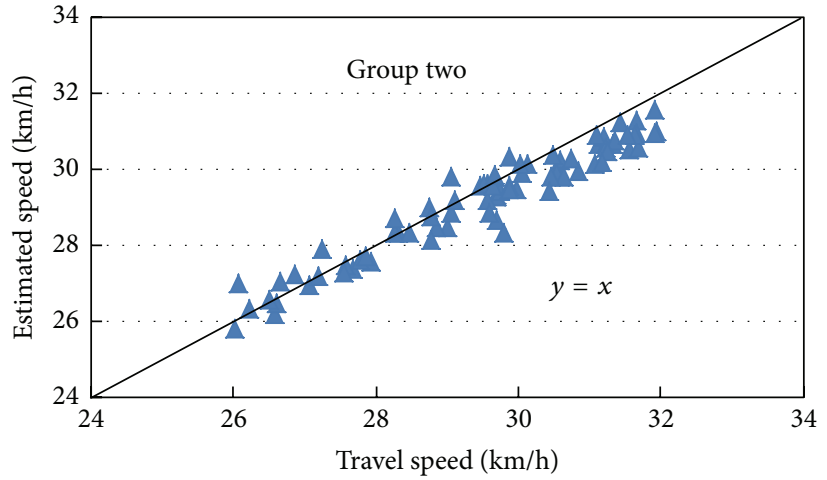

(b)

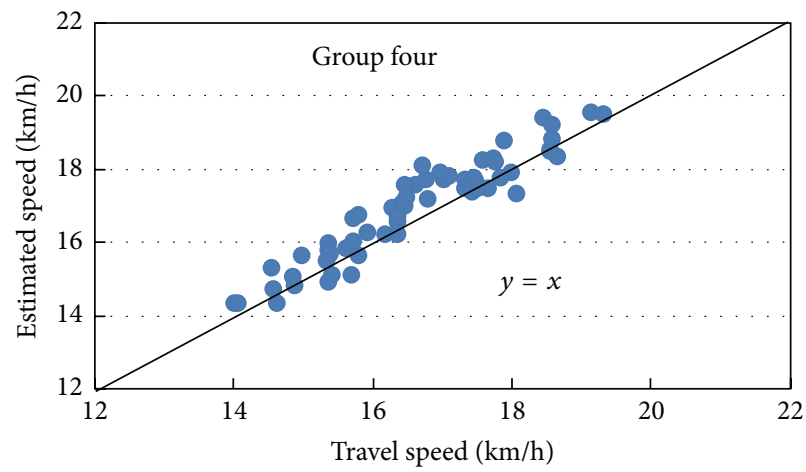

(d)

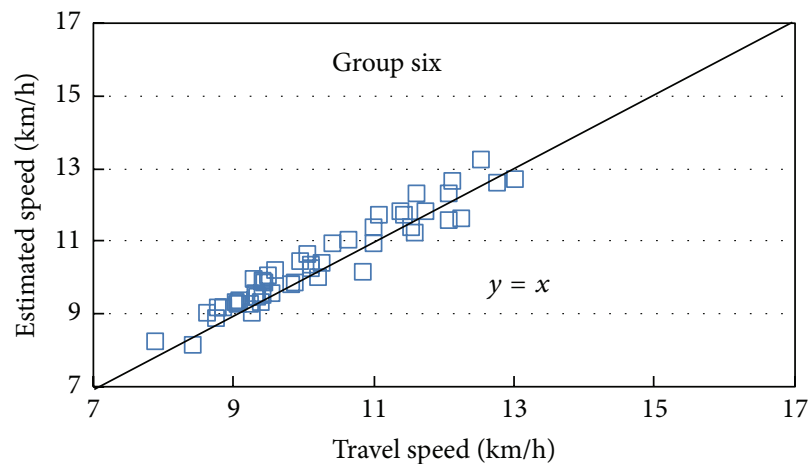

(f)

FIGURE 7: Average travel speed estimation results.

If the vehicles continue to enter this urban region, the traffic condition would be worse, and the traffic volume and travel speed would both decrease. In fact, to maintain a relative suitable service level, the points cannot be permitted to reach the maximum point. Therefore, during the peak period, the right side of the maximum point is suitable for an urban region to maintain by means of border control and so forth. With regard to the travelers, they can choose their routes rapidly or estimate the cost in terms of travel time by using the information of average travel speed of an urban region.

\section{Conclusion and Discussion}

To evaluate traffic conditions in an urban region quantitatively and to offer a feasible method to guide the traffic managers and travelers, we select the average travel speed in an urban region network and average traffic flow per lane. As it is difficult to collect the traffic parameter of travel speed from widely distributed fixed detectors directly, we calculate travel speed with a traffic parameter, which can be measured by traffic data from fixed detectors. The relationship between average travel speed and queue lengths at signalized intersections within the urban area is established based on a mathematical assumption. Finally, the average estimated travel speed model proposed in this paper is tested with microscopic simulation platform, and the results showed that the average estimated travel speed model performed well with satisfactory accuracy, which means that this method is helpful and can reduce the cost of placing detectors. The best performance of the network efficiency can be identified based 


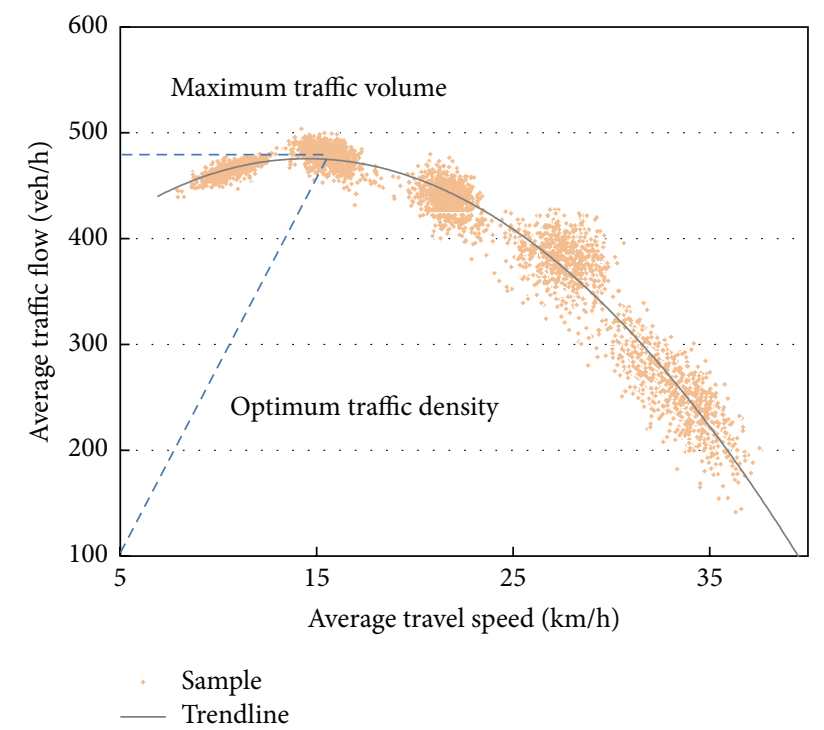

FIGURE 8: Macroscopic fundamental diagram for urban region network.

on the macroscopic fundamental diagram, and the method is simple enough for application in engineering. Therefore, the result is instructive for creating both traffic control strategies and route choices. However, the limitations are listed as follows.

(1) The real-time maximum queue lengths are output from simulation software instead of being calculated by traditional methods (e.g., Liu et al., 2009) [14]. Although the estimation model of travel speed performs well in calculating average travel speed in an urban region network, it also depends on the accuracy of the model for maximum queue length estimation, which makes the proposed system evaluate traffic state less accurately.

(2) As there are different characteristics among drivers, the free travel speed is a distribution instead of a stable value.

(3) We will conduct more experiments to test the performance of the proposed model on real field test platforms. There are some other problems we will meet, such as errors caused by detectors. Therefore, a pretreatment is necessary to deal with the raw data collected by fixed detectors.

\section{Conflict of Interests}

The authors declare that there is no conflict of interests regarding the publication of this paper.

\section{Acknowledgment}

The work was supported by the National Natural Science Foundation of China (Grant no. 51378237).

\section{References}

[1] Y. J. Stephanedes and G. Vassilakis, "Intersection incident detection for IVHS," in Proceedings of the Transportation Research Board 74th Annual Meeting, Washington, DC, USA, 1994.
[2] M. G. H. Bell and B. Thancanamootoo, "Automatic incident detection within urban traffic control systems," in Proceedings of the International Road and Traffic Conference Roads, vol. 4, pp. 35-39, Berlin, Germany, September 1988.

[3] L. D. Han and A. D. May, "Automatic detection of traffic operational problems on urban arterials," Tech. Rep., Institute of Transportation Studies, University of California, 1989.

[4] K. Thomas, H. Dia, and N. Cottman, "Simulation of arterial incident detection using neural networks," in Proceedings of the 8th World Congress on Intelligent Transport Systems, Sydney, Australia, 2001.

[5] Q. Zhao, Q.-J. Kong, Y. Xia, and Y. Liu, "Sample size analysis of GPS probe vehicles for urban traffic state estimation," in Proceedings of the 14th IEEE International Intelligent Transportation Systems Conference (ITSC '11), pp. 272-276, IEEE, Washington, DC, USA, October 2011.

[6] B. S. Kerner, C. Demir, R. G. Herrtwich et al., "Traffic state detection with floating car data in road networks," in Proceedings of the IEEE Intelligent Transportation Systems Conference, pp. 44-49, IEEE, 2005.

[7] A. Lozano, G. Manfredi, and L. Nieddu, "An algorithm for the recognition of levels of congestion in road traffic problems," Mathematics and Computers in Simulation, vol. 79, no. 6, pp. 1926-1934, 2009.

[8] S. Tao, V. Manolopoulos, S. Rodriguez, and A. Rusu, "Realtime urban traffic state estimation with A-GPS mobile phones as probes," Journal of Transportation Technologies, vol. 2, no. 1, pp. 22-31, 2012.

[9] H. Dia and K. Thomas, "Development and evaluation of arterial incident detection models using fusion of simulated probe vehicle and loop detector data," Information Fusion, vol. 12, no. 1, pp. 20-27, 2011.

[10] Q.-J. Kong and Y.-C. Liu, "Model of federated evidence fusion for real-time urban traffic state estimation," Journal of Shanghai Jiaotong University (Science), vol. 12, no. 6, pp. 793-804, 2007.

[11] M. J. Lighthill and G. B. Whitham, "On kinematic waves. I. Flood movement in long rivers," Proceedings of the Royal Society, vol. 229, pp. 281-316, 1955.

[12] P. I. Richards, "Shock waves on the highway," Operations Research, vol. 4, no. 1, pp. 42-51, 1956.

[13] P. G. Michalopoulos and G. Stephanopoulos, "Oversaturated signal systems with queue length constraints: single intersection," Transportation Research, vol. 11, no. 6, pp. 413-421, 1977.

[14] H. X. Liu, X. Wu, W. Ma, and H. Hu, "Real-time queue length estimation for congested signalized intersections," Transportation Research Part C: Emerging Technologies, vol. 17, no. 4, pp. 412-427, 2009.

[15] C. F. Daganzo and N. Geroliminis, "An analytical approximation for the macroscopic fundamental diagram of urban traffic," Transportation Research Part B: Methodological, vol. 42, no. 9, pp. 771-781, 2008.

[16] N. Geroliminis and C. F. Daganzo, "Existence of urban-scale macroscopic fundamental diagrams: some experimental findings," Transportation Research Part B: Methodological, vol. 42, no. 9, pp. 759-770, 2008. 


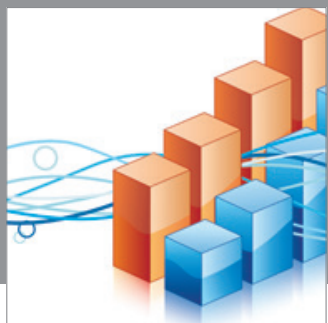

Advances in

Operations Research

mansans

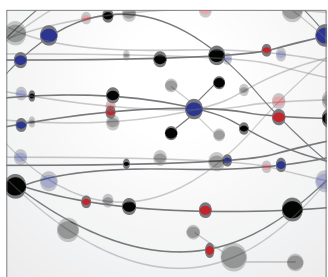

The Scientific World Journal
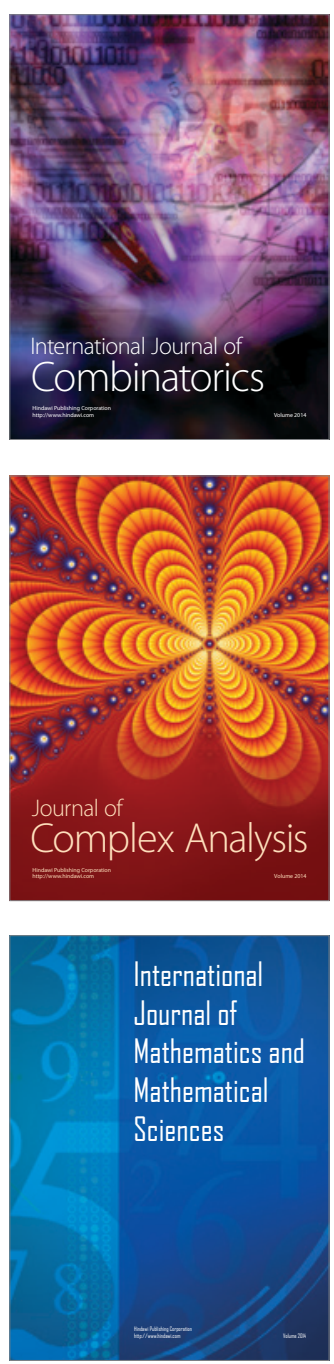
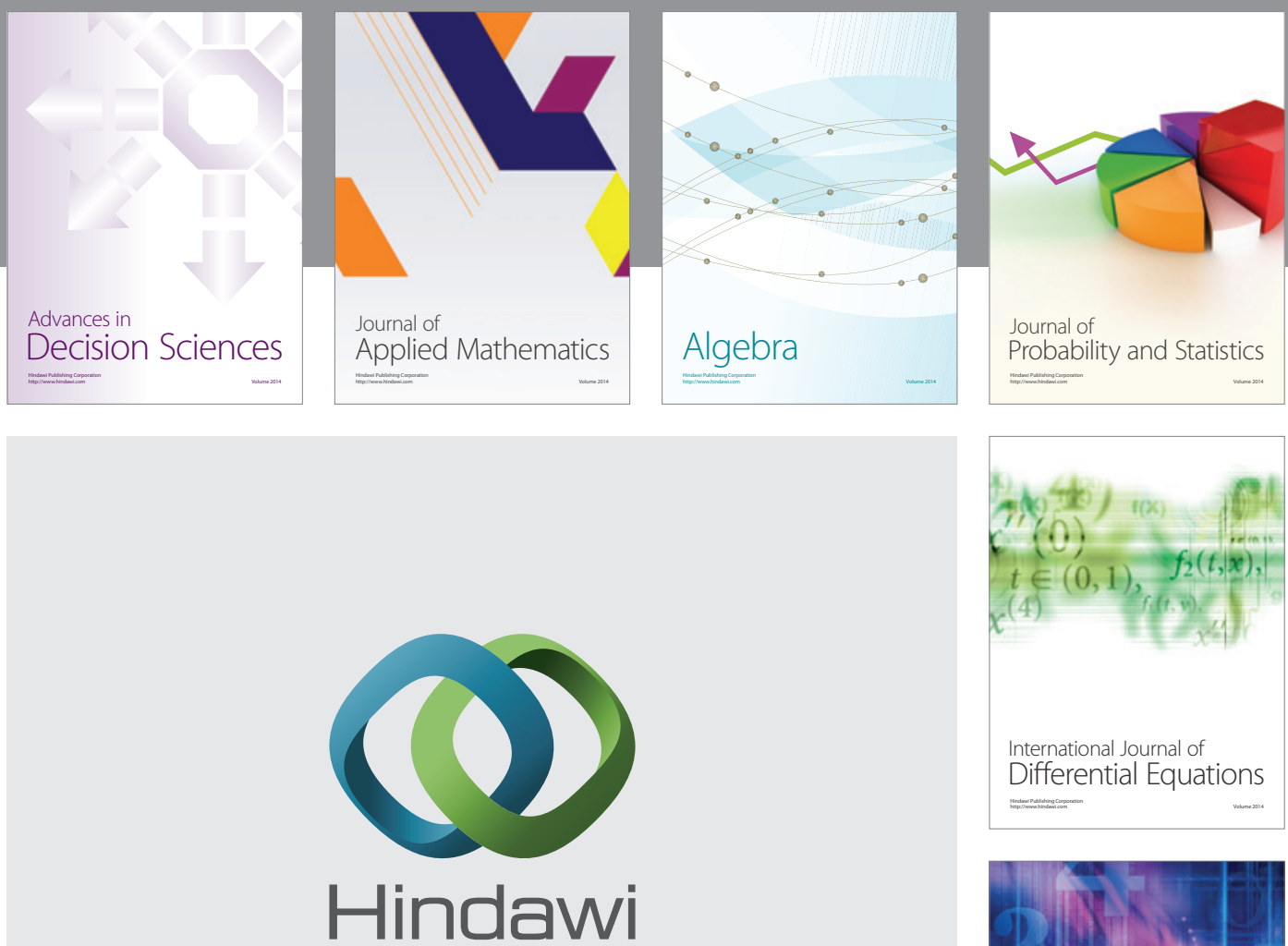

Submit your manuscripts at http://www.hindawi.com
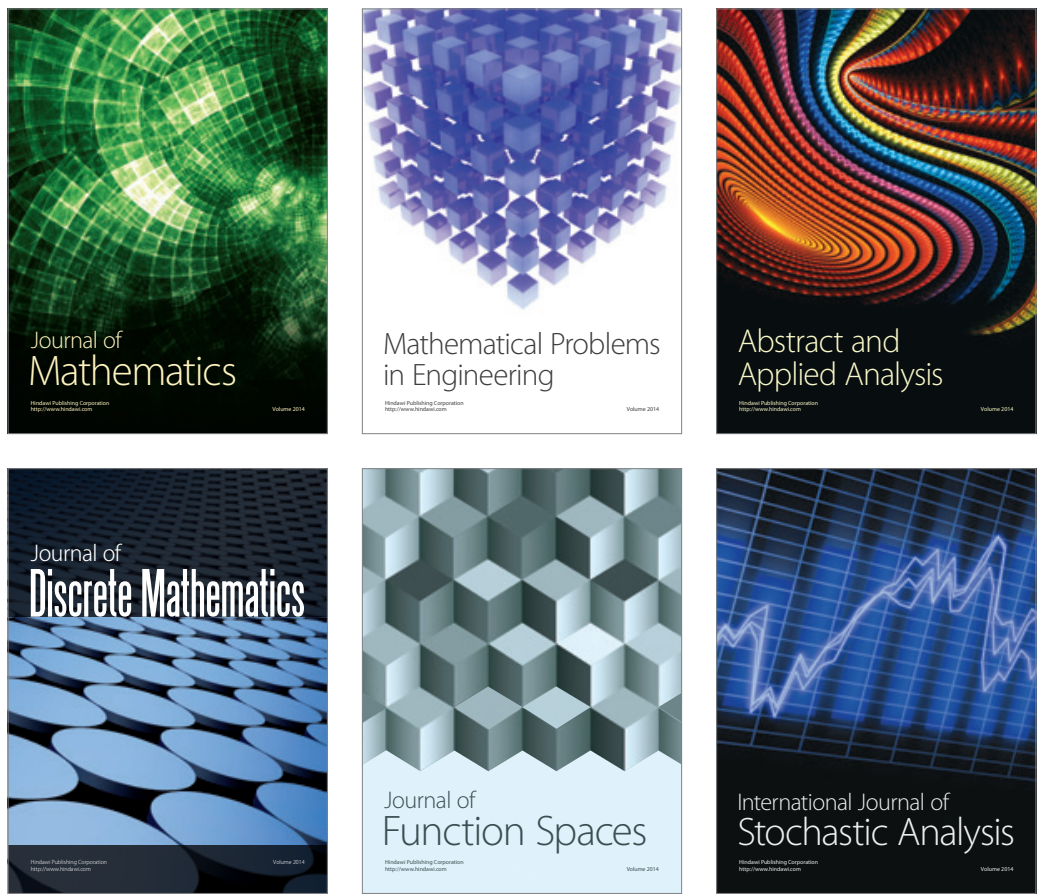

Journal of

Function Spaces

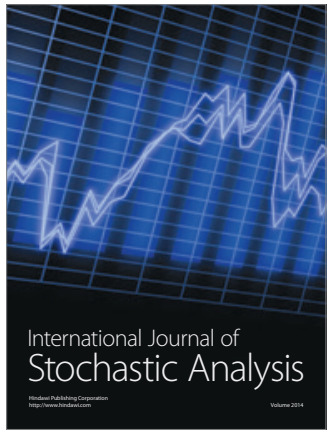

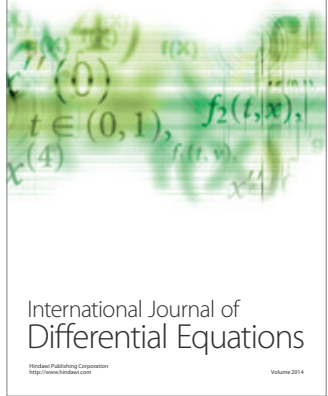
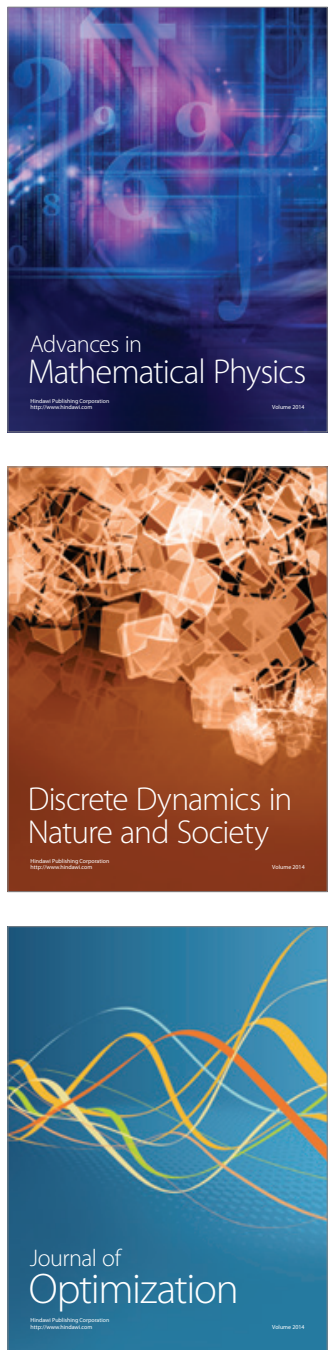